\title{
Study on Helium Bubble Coalescence in Titanium with Dislocations
}

\author{
Baoling Zhang*, Xue Su, Chongyang Li, Junwei Zhang \\ North China University of Water Resources and Electric Power, Zhengzhou, China \\ Email: ${ }^{\star}$ zbaoling1234@163.com
}

How to cite this paper: Zhang, B.L., Su, X., Li, C.Y. and Zhang, J.W. (2021) Study on Helium Bubble Coalescence in Titanium with Dislocations. World Journal of Nuclear Science and Technology, 11, 73-83. https://doi.org/10.4236/wjnst.2021.112005

Received: February 18, 2021

Accepted: March 29, 2021

Published: April 1, 2021

Copyright (C) 2021 by author(s) and Scientific Research Publishing Inc. This work is licensed under the Creative Commons Attribution International License (CC BY 4.0).

http://creativecommons.org/licenses/by/4.0/

\begin{abstract}
Dislocation and grain boundary have great influence on helium behavior in materials. In this paper, the helium bubble coalescence in titanium with dislocations was simulated using molecular dynamics method. The results show that, when the second helium bubble nucleates near the slip plane, it grows toward the first helium bubble which lies at the dislocation core till they coalesce with each other. However, it is not easy for the coalescence to occur if the two helium bubbles lie in different atomic layers in (001) plane. If the second helium bubble is nucleated on the side of the slip plane with full atomic layers, the second helium bubble growth could lead to the movement of the first helium bubble toward the other sides of the slip plane. The growth rate and direction of the second helium bubble are closely related to the pressure around it.
\end{abstract}

\section{Keywords}

Helium Bubble, Coalescence, Dislocation, Molecular Dynamics Simulation

\section{Introduction}

In fusion equipment Tokamak, the first wall has to withstand very harsh conditions. One of particular concerns is the serious radiation damage of materials induced by the helium particles escaping from the plasma or $(n, \alpha)$ reaction [1] [2]. It has already been proven that helium atoms prefer to migrate and gather forming helium bubbles which can seriously destroy the mechanical properties of materials, such as the material deformation, cracking, embrittlement, and so on. It is crucial to improve the ability of materials to resist helium irradiation damage in the development of nuclear fusion.

Many experiments have been carried out in order to improve the helium resistance ability of materials [3] [4] [5]. Fabritsiev et al. [3] studied the effect of 
the grain size and high rate of helium accumulation on radiation resistance of pure copper for fusion equipment applications. It is found that grain size has obvious effects on the hardening and the loss of ductility of pure copper. In the study of Jeuland et al. [4], the high density of grain boundaries in the finer microstructure explains the thermal shift and the larger desorption at low temperature. It is obvious that dislocation and grain boundary have great influence on helium behavior in materials. Titanium is an important nuclear material, however, the helium bubble coalescence in titanium with dislocations is still unclear.

Molecular Dynamics (MD) simulation is a powerful tool for studying helium behavior in materials [6] [7]. In this paper, the influence of dislocations and grain boundary on helium bubble coalescence is studied using MD method. Titanium was used as substrate because of a series of excellent properties, such as high temperature and corrosion resistance, titanium alloy with high strength and light texture. Moreover, titanium also refines the grain size and improves the strength, plasticity and toughness of metals [8] [9]. Symmetrical inclined small angle grain boundary was constructed in titanium substrate. Some new interaction mechanisms which are beneficial to improve the ability of materials to resist helium irradiation damage were found.

\section{Simulation Method}

In the following Molecular Dynamics (MD) simulation, all the atoms of the system obey the Newton equation of motion. The integration method is leap-frog algorithm and the integration step is set to $1 \mathrm{fs}$.

The MD simulation results depend strongly on the interaction potential adopted. In this paper, the Lennard-Jones was adopted to describe the interactions between helium atoms because it has been proved to be well suited to the properties of noble gases [10]. The Ti-Ti interaction was described by tight-binding potential obtained by Cleri and Rosato [11]. As for the interaction between titanium and helium atoms, Wang et al. [12] constructed the Ti-He potential by fitting the pair potential to ab initio data, which is written as follows,

$$
V(r)=\varepsilon\left[\left(\frac{r_{0}}{r}\right)^{4.35}-3.21\left(\frac{r_{0}}{r}\right)^{1.4}\right]
$$

where $\varepsilon=0.02617 \mathrm{eV}, r_{0}=0.47288 \mathrm{~nm}, r$ denotes the distance between $\mathrm{He}$ and $\mathrm{Ti}$ atoms. The availability of the above potentials has been proven in our former simulations which gave reasonable simulation results [13] [14].

The titanium substrate contains dislocations and surface, which was constructed as follows. The substrate was made up of two parts. One part had the full atomic layers $N$ and the other part was $N-1$ layers. The two parts had the same width, and so the crystal planes of the two parts contained a dislocation. The method was proposed by Daw and Baskes, which has been proved to be very successful in the studies of edge dislocations [15] [16] [17]. In addition, symmetrical inclined small angle grain boundary was composed of a series of 
parallel edge dislocations. The periodic boundary conditions were applied to the direction of the Boggs vector and also to the direction of the dislocation. The third direction contained the metal surface. The substrate contains 34,050 titanium atoms. The simulation temperature is in the range of $0-500 \mathrm{~K}$ and the time step of the integral is $1 \mathrm{fs}$.

In the simulations, the first helium bubble was formed in the dislocation core by introducing helium atoms one by one near the dislocation core, as shown in Figure 1. Then the other helium bubble was also formed by introducing helium atoms one by one inside A and side B respectively, which was named the second helium bubble. The growth and coalescence of the second helium bubble in different sides of the slip plane as well as the influence on the substrate were simulated and compared in detail.

In order to observe clearly the behaviors of helium atoms and the substrate evolution induced by the interaction between atoms and dislocations. It is better to avoid the temperature-induced defects. Therefore, the simulation temperature was firstly set to $0 \mathrm{~K}$. Then the temperature of the Ti-He system was increased using electron-phonon coupling model, in which the atoms exchange energy with the electron gas in the substrate according to the electron-phonon interaction dynamics [18].

In addition, for convenience in the following analysis, the atomic layers containing the helium bubbles, i.e., the atomic layers in the solid line box in Figure 1 , are cut out and displayed.

\section{Results and Discussions}

\subsection{Helium Bubble Coalescence in Side A}

In Figure 2, the second helium bubble is nucleated at about three lattice units from the first helium bubble. The two helium bubbles lie in the same titanium atomic layers in (001) plane. It can be seen that the second helium bubble grows toward the first helium bubble, however, the position of the lower boundary of the helium bubble is almost constant. The position of the upper boundary of the helium bubble moves about $0.24 \mathrm{~nm}$ within $23 \mathrm{ps}$, accordingly, the migrating rate toward the slip plane is about $10.4 \mathrm{~nm} / \mathrm{ns}$. When the addition number of

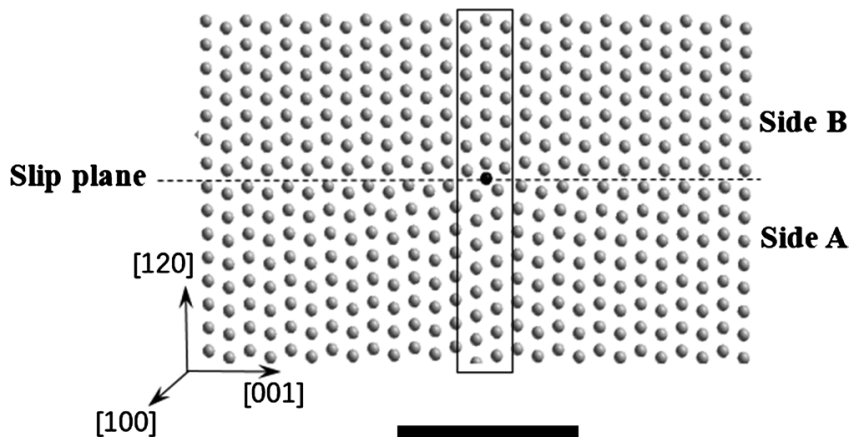

Figure 1. The first helium atom introduced within the first helium bubble in the dislocation core. The dot line in figure is the slip plane, the same below. 



Figure 2. The growth process of the second helium bubble at the same atomic layers in (001) plane with the first helium bubble. The number of helium atoms within the second helium bubble is 1,5 , and 7 respectively.

helium atoms attain to 7 , coalescence occurs between each other as shown in Figure 2(c). During the coalescence process, all the helium atoms within the second bubble migrate rapidly toward the first helium bubble, and the coalesced helium bubble lies finally in the dislocation core. This fully indicates that the dislocation core has a stronger ability to capture helium bubbles because of the larger binding energy of helium with dislocations and grain boundaries.

In Figure 3, the second helium bubble is also nucleated near the first helium bubble, however, the two helium bubbles no longer lie in the same (001) plane. It is found that the second helium bubble grows toward the slip plane, at the same time, it also grows towards the [ $00 \overline{1}]$ direction till it attains to the pre-existing grain boundary, as shown in Figure 3(b) and Figure 3(c). The coalescence of the two helium bubbles has not been observed even if the number of helium atoms within the second helium bubble reaches 200, as shown in Figure 3(d). Higher temperature is benefit for the migration of atoms, so the temperature of the system is increased to $300 \mathrm{~K}$. The second helium bubble is observed to expand toward the slip plane. However, it is still not observed the helium bubbles coalescence. The result indicates that, in contrast to the helium bubbles lying in the same atomic layer, it is not easy for the coalescence to occur of the two helium bubbles lying in different (001) plane. In the study of Chen et al. [19], single helium atom, He-dimer and He-trimer tend to jump between layers along [001] direction. However, the inter-layer jump along [001] direction seems to be difficult after helium bubble grows up in the simulation.

Figure 4 shows the influence of the helium bubble growth near the grain 

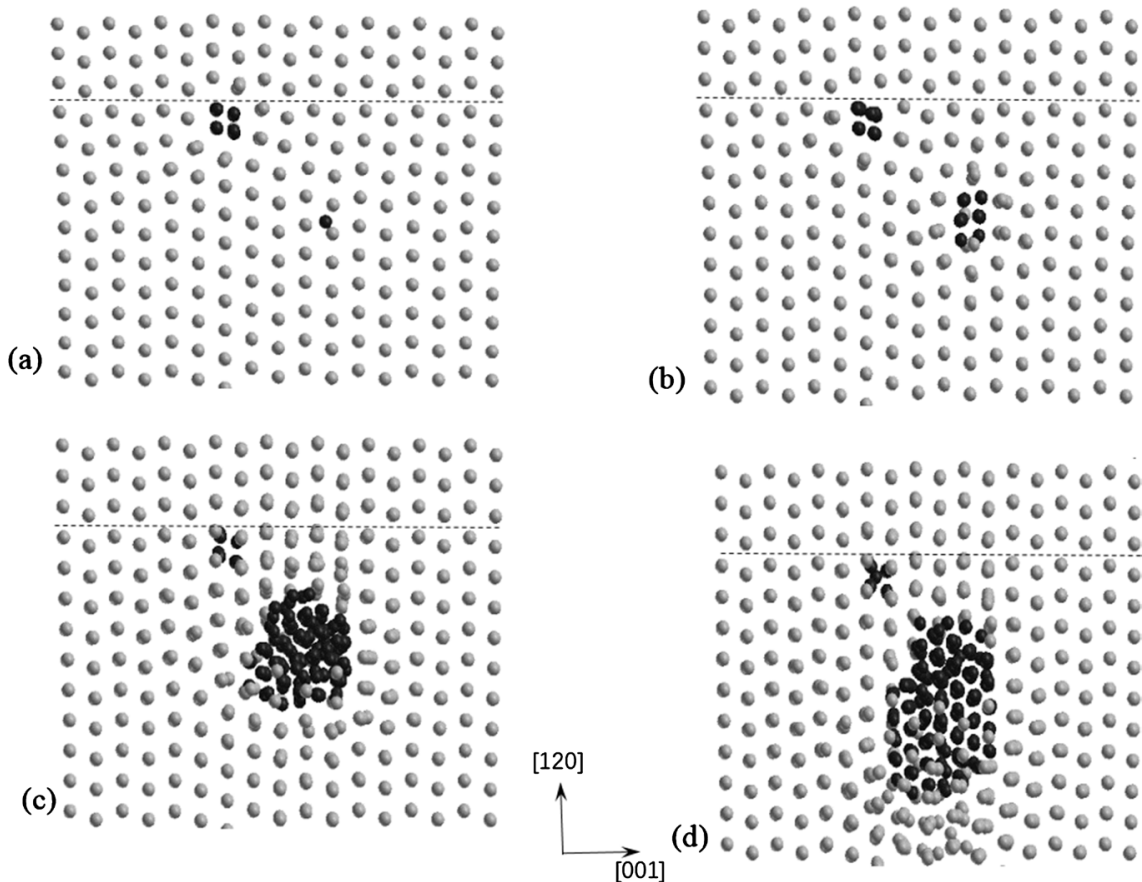

Figure 3. The growth process of the second helium bubble at the different atomic layers in (001) plane with the first helium bubble. The number of helium atoms within the second helium bubble is (a) 1, (b) 10, (c) 100 and (d) 200, respectively.

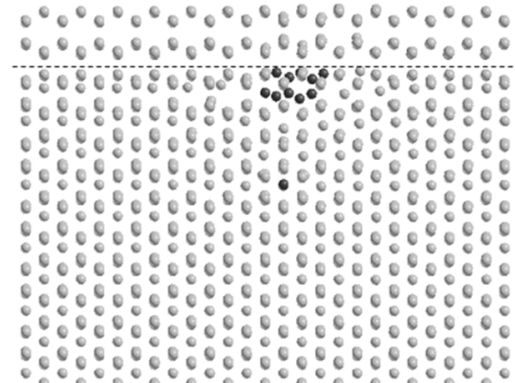

(a)

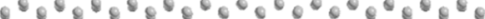

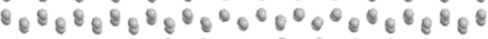
$080808088^{2}$

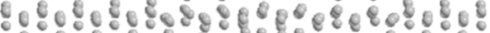

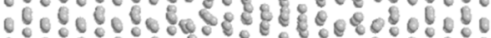

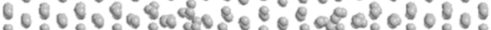

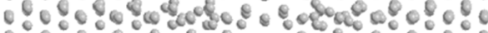





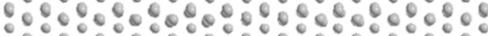

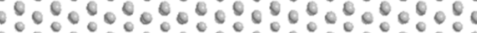

(b)

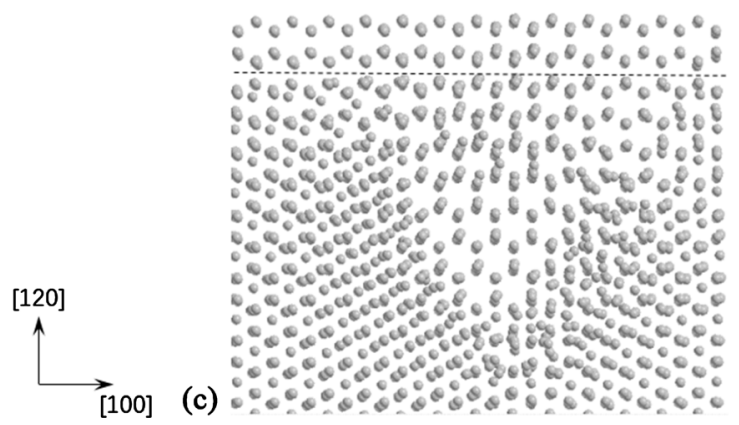

Figure 4. The influence of the helium bubble growth near the grain boundary on the substrate. The second helium bubble contains (a) 1, (b) 50 and (c) 200 helium atoms respectively. The helium atoms are hidden in (b) and (c).

boundary on the substrate. In our former studies, the pressure within helium bubble in titanium has been calculated which is GPa in order magnitude [20]. Because of the high pressure within the helium bubble, some pre-existing misa- 
ligned metal atoms are pushed to the lattice sites in the simulation. The crystal structure is partly recovered around the helium bubbles. However, defects increase in the adjacent region of the helium bubbles.

Then the influence of the metal surface on the growth rate and direction of the second helium bubble is studied. The initial distance between the second helium bubble and the metal surface is $10.8,8,4.5$ and 2 lattice units respectively. The dependence of the helium bubble growth rate on the distance is shown in Table 1 . When the distance from the metal surface is larger than 8 lattice units, the helium bubble grows only toward the slip plane. When the distance is 4.5 lattice units, it grows toward both the slip plane and the metal surface, and the growth rate toward the metal surface is larger than the growth rate toward the slip plane. When the distance is 2 lattice units, helium release occurs. It can be found that, as the distance from the metal surface decreases, the helium bubble growth rate increases toward the metal surface and decreases sharply toward the slip plane. Therefore, the growth rate and direction of helium bubble strongly depend on the distance between the helium bubble and the slip plane or the metal surface.

As for the influence of helium bubble growth on the substrate, it depends strongly on the position of helium bubble. If the slip plane is closer to the helium bubble in comparison of the metal surface, defects prefer to produce on the side near the slip plane, as shown in Figure 5(a). On the contrary, when the metal surface is closer to the helium bubble, defects prefer to produce on the side near the metal surface and migrate toward the metal surface, leading to the increasing number of surface metal atoms and the increasing surface roughness, as shown in Figure 5(b).

\subsection{Helium Bubble Coalescence in Side B}

In the following simulation, the second helium bubble is nucleated at about 3.2 lattice units from the first helium bubble, however, it lies in side B which has the full metal atomic layers, as shown in Figure 6(a). It is found that the helium bubble also grows toward the slip plane with the addition of helium atoms as shown in Figure 6(b). Because of the high pressure within the helium bubble, the pre-existing misaligned metal atoms are pushed to the lattice sites, and the crystal lattice around the first helium bubble is partly restored. The recovery of the crystal structure near the slip plane causes the movement of the first helium bubble about one lattice toward [ $1 \overline{2} 0$ ] direction, as shown in Figure 7.

Table 1. Helium bubble growth rate in side A.

\begin{tabular}{cccc}
\hline \multicolumn{2}{c}{ Distance (lattice unit) } & \multicolumn{2}{c}{ Growth rate (nm/ns) } \\
\hline Fromthe slip plane & From themetal surface & toward the slip plane & toward the metal surface \\
\hline 3.2 & 10.8 & 10 & 0 \\
6 & 8 & 4.9 & 0 \\
9.5 & 4.5 & 0.98 & 1.5 \\
12 & 2 & 0 & Helium release
\end{tabular}



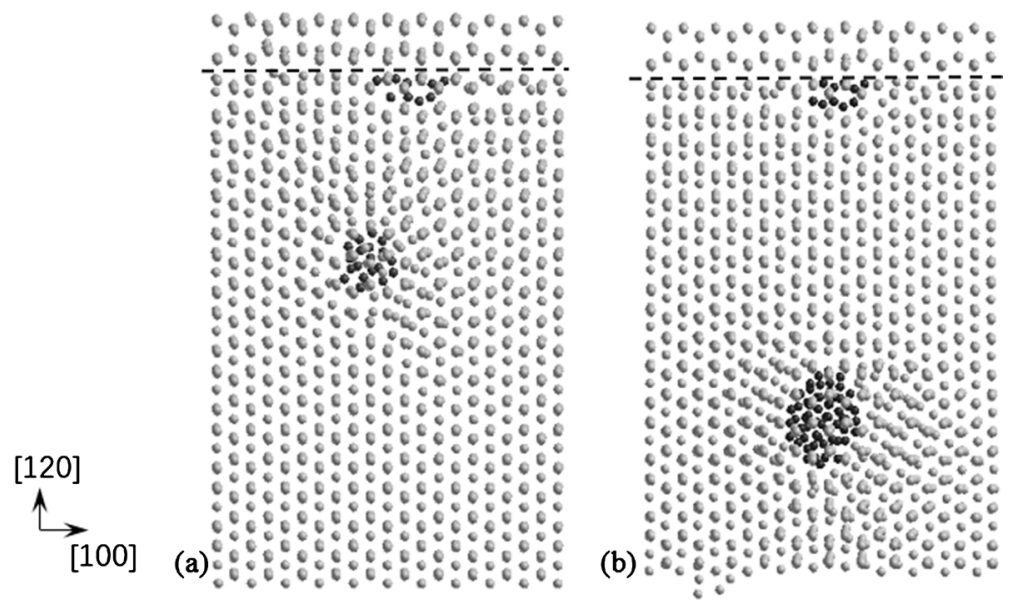

Figure 5. The defects induced by helium bubble growth. (a) The second helium bubble is closer to the slip plane, (b) The second helium bubble is closer to the metal surface.
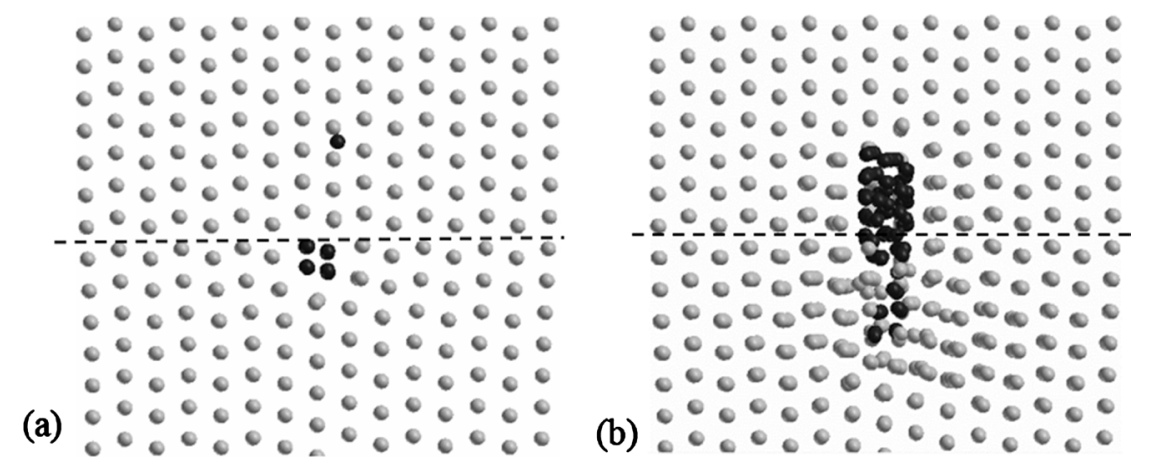

Figure 6. The growth of the second helium bubble nucleated in side B. (a) The initial position of the second helium bubble, (b) The second helium bubble growing toward the slip plane.
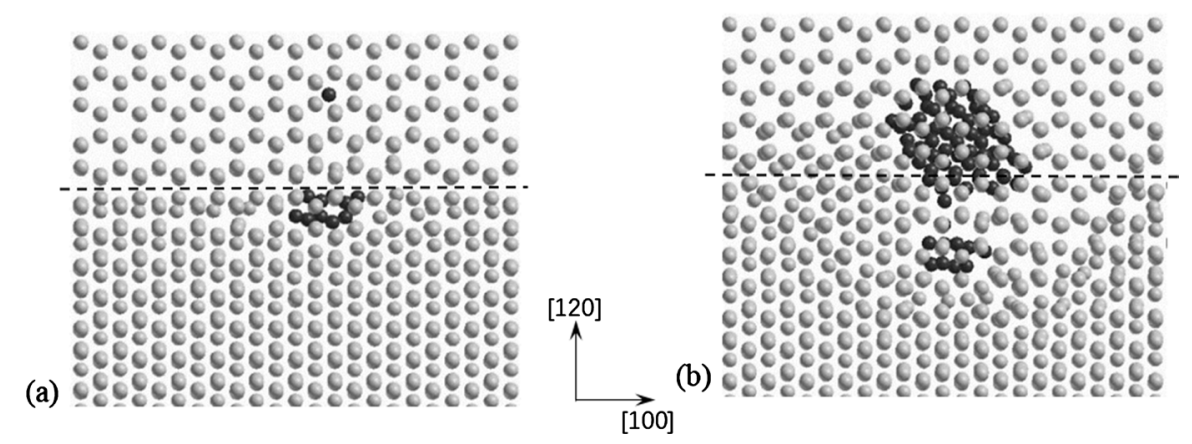

Figure 7. The movement of the first helium bubble. (a) The initial position of the first helium bubble, (b) The movement of the first helium bubble toward [1 $\overline{2} 0$ ] direction.

When the number of the addition helium atoms attain to 63, coalescence occurs between the first and the second helium bubble. Then the coalesced helium bubble grows continually toward [1 $\overline{2} 0$ ] direction. During the helium bubble growing and coalescence process, defects prefer to produce near the slip plane as shown in Figure 8.

Then the influence of temperature on the behavior of the helium bubble and 
the substrate is observed. Keeping the number of helium atoms constant, the temperature of the system is increased to $300 \mathrm{~K}$. At room temperature, the coalesced helium bubble inclines to expand outward in (001) plane along the slip plane, but it is difficult for the helium bubble expansion through the atomic layers of the substrate in [001] direction, as shown in Figure 9.

The influence of the metal surface on the growth rate and direction of the second helium bubble is also studied. The dependence of the helium bubble growth rate on the distance is shown in Table 2. As the helium bubble gets closer to the metal surface, the helium bubble growth rate toward the metal surface increases, which is the same as the simulation result of side A. When the helium bubble is equidistant from the slip plane and the metal surface, it grows faster

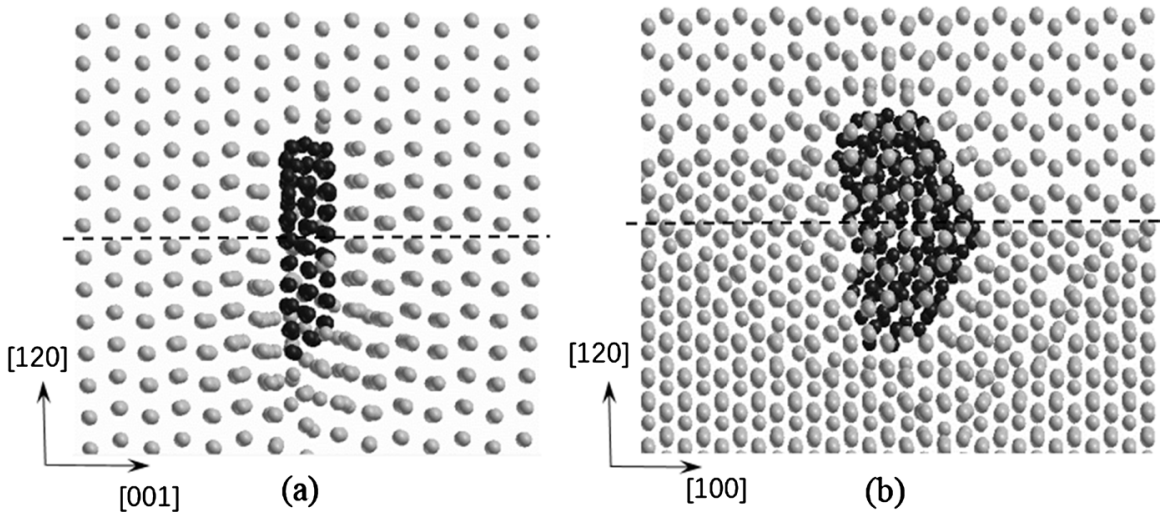

Figure 8. The substrate and the second helium bubble at 0 K. (a) (100) plane, (b) (001) plane.
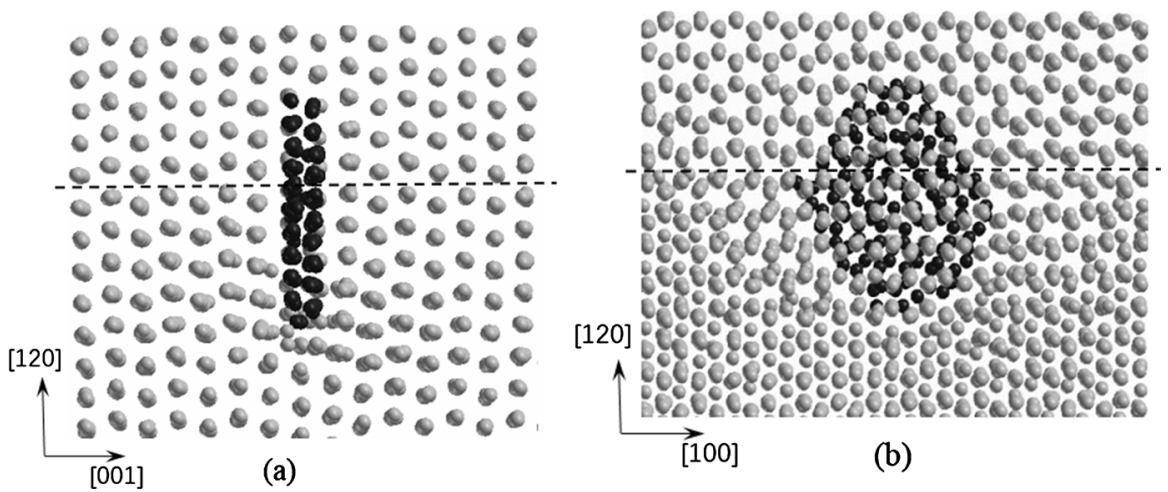

Figure 9. The substrate and the second helium bubble at $300 \mathrm{~K}$. (a) (100) plane, (b) (001) plane.

Table 2. Helium bubble growth rate in side B.

\begin{tabular}{cccc}
\hline \multicolumn{2}{c}{ Distance (lattice unit) } & \multicolumn{2}{c}{ Growth rate (nm/ns) } \\
\hline From the slip plane & from themetal surface & toward the slip plane & toward the metal surface \\
\hline 3.7 & 9.3 & 3.03 & 0 \\
5 & 8 & 2.57 & 0 \\
8 & 5 & 0 & 3.63 \\
\hline
\end{tabular}


toward the metal surface. For example, when the distance is 5 lattice unit, the growth rate of the helium bubble is $2.57 \mathrm{~nm} / \mathrm{ns}$ toward the slip plane and 3.63 $\mathrm{nm} / \mathrm{ns}$ toward the metal surface respectively. In addition, the growth rate of helium bubble in side B is smaller than that in side A. All the former simulation results show that the growth rate and direction of the second helium bubble are closely related to the pressure around it. Helium bubble always grows toward the direction with lower pressure, and the lower the pressure, the faster it grows.

\section{Conclusion}

The helium bubble coalescence in titanium containing dislocations and grain boundary was studied using molecular dynamics method. When the second helium bubble is nucleated on one side of the slip plane, it always grows toward the slip plane. If the two helium bubbles lie in the same titanium atomic layers in (001) plane, they coalesce easily and the coalesced helium bubble lies in the dislocation core. In addition, the pre-existing misaligned metal atoms could be pushed to the lattice sites because of the high pressure within the second helium bubble. The recovery of the crystal structure near the slip plane causes the movement of the first helium bubble toward the side which is short of one atomic layer. The influence of the metal surface on the growth rate and direction of the second helium bubble is also studied. As the initial distance between the second helium bubble and the metal surface decreases, the helium bubble growth rate toward the surface increases and accordingly it decreases sharply toward the slip plane. The growth rate and direction of helium bubble are closely related to the pressure around the helium bubble.

\section{Funding}

This work was supported by the Key Scientific Research Project in Colleges and Universities of Henan Province, China (Grant Nos. 20A490001).

\section{Conflicts of Interest}

The authors declare no conflicts of interest regarding the publication of this paper.

\section{References}

[1] Rowcliffe, A.F., Garrison, L.M., Yamamoto, Y., Tan, L. and Katoh, Y. (2018) Materials Challenges for the Fusion Nuclear Science Facility. Fusion Engineering and Design, 135, 290-301. https://doi.org/10.1016/j.fusengdes.2017.07.012

[2] Rieth, M., Doerner, R., Hasegawa, A., Ueda, Y. and Wirtz, M. (2019) Behavior of Tungsten under Irradiation and Plasma Interaction. Journal of Nuclear Materials, 519, 334-368. https://doi.org/10.1016/j.jnucmat.2019.03.035

[3] Fabritsiev, S.A. and Pokrovsky, A.S. (2003) The Effects of Grain Size and Helium Accumulation on Radiation Hardening and Loss of Ductility of Pure Copper for ITER Application. Fusion Engineering and Design, 65, 545-559. https://doi.org/10.1016/S0920-3796(03)00314-4 
[4] Jeuland, H.L., Moll, S., Jourdan, T. and Legendre, F. (2013) Effect of Grain Microstructure on Thermal Helium Desorption from Pure Iron. Journal of Nuclear Materials, 434, 152-157. https://doi.org/10.1016/j.jnucmat.2012.11.025

[5] Sakaguchi, N., Ohguchi, Y., Shibayama, T., Watanabea, S. and Kinoshitab, H. (2013) Surface Cracking on $\Sigma 3, \Sigma 9$ CSL and Random Grain Boundaries in Helium Implanted 316L Austenitic Stainless Steel. Journal of Nuclear Materials, 432, 23-27. https://doi.org/10.1016/j.jnucmat.2012.08.019

[6] Grigoreva, P., Bakaev, A., Terentyev, D., Oost, G.V., Noterdaeme, J.M. and Zhurkinc, E.E. (2017) Interaction of Hydrogen and Helium with Nanometric Dislocation Loops in Tungsten Assessed by Atomistic Calculations. Nuclear Instruments and Methods in Physics Research Section B: Beam Interactions with Materials and Atoms, 393, 164-168. https://doi.org/10.1016/j.nimb.2016.10.036

[7] Fu, J., Chen, Y.C., Fang, J.Z., Gao, N., Hu, W.Y., Jiang, C., Zhou, H.B., Lv, G.H., Gao, F. and Deng, H.Q. (2019) Molecular Dynamics Simulations of High-Energy Radiation Damage in W and W-Re Alloys. Journal of Nuclear Materials, 524, 9-20. https://doi.org/10.1016/j.jnucmat.2019.06.027

[8] Wang, J.C., Wang, W.J., Wei, R., Wang, X.L., Sun, Z.X., Xie, C.Y., Li, Q. and Luo, G.N. (2017) Effect of Ti Interlayer on the Bonding Quality of W and Steel HIP Joint. Journal of Nuclear Materials, 485, 8-14. https://doi.org/10.1016/j.jnucmat.2016.12.024

[9] Buzi, L., Yeh, M., Yeh, Y.W. and Donaldson, O.K. (2019) Deuterium and Helium Ion Irradiation of Nanograined Tungsten and Tungsten-Titanium Alloys. Nuclear Materials and Energy, 21, Article ID: 100713. https://doi.org/10.1016/j.nme.2019.100713

[10] Donnelly, S.E. and Evans, J.H. (1991) Fundamentals Aspects of Inert Gases in Solid. Plenum, New York. https://doi.org/10.1007/978-1-4899-3680-6

[11] Cleri, F. and Rosato, V. (1993) Tight-Binding Potentials for Transition Metals and Alloys. Physical Review B, 48, 22. https://doi.org/10.1103/PhysRevB.48.22

[12] Wang, J., Hou, Q., Sun, T.Y., Wu, Z.C., Long, X.G., Wu, X.C. and Luo, S.Z. (2006) Simulation of Helium Behaviour in Titanium Crystals Using Molecular Dynamics. Chinese Physics Letters, 23, 1666.

[13] Zhang, B.L., Wang, J., Li, M. and Hou, Q. (2013) A Molecular Dynamics Study of Helium Bubble Formation and Gas Release near Titanium Surfaces. Journal of Nuclear Materials, 438, 178-182. https://doi.org/10.1016/j.jnucmat.2013.03.033

[14] Wang, J., Hou, Q., Sun, T.Y., Long, X.G., Wu, X.C. and Luo, S.Z. (2007) Defect Behavior Induced by Helium Cluster Growth in Titanium Crystals. Journal of Applied Physics, 102, Article ID: 093510. https://doi.org/10.1063/1.2804110

[15] Rodney, D. and Martin, G. (1999) Dislocation Pinning by Small Interstitial Loops: A Molecular Dynamics Study. Physical Review Letters, 82, 3272.

https://doi.org/10.1103/PhysRevLett.82.3272

[16] Osetsky, N.Y. and Bacon, D.J. (2003) An Atomic-Level Model for Studying the Dynamics of Edge Dislocations in Metals. Modelling and Simulation in Materials Science and Engineering, 11, 427. https://doi.org/10.1088/0965-0393/11/4/302

[17] Zhang, B.L., Wang, B.W., Su, X., Song, X.Y. and Li, M. (2018) Growth Mode of Helium Crystal near Dislocations in Titanium. Chinese Physics B, 27, Article ID: 060205. https://doi.org/10.1088/1674-1056/27/6/060205

[18] Hou, Q., Hou, M., Bardotti, L., Prével, B., Mélinon, P. and Perez, A. (2000) Deposition of Au N Clusters on $\mathrm{Au}(111)$ Surfaces. I. Atomic-Scale Modeling. Physical Re- 
view $B$, 62, 2825. https://doi.org/10.1103/PhysRevB.62.2825

[19] Chen, M., Hou, Q., Wang, J., Sun, T.Y., Long, X.G. and Luo, S.Z. (2008) Anisotropic Diffusion of He in Titanium: A Molecular Dynamics Study. Solid State Communications, 148, 178-181. https://doi.org/10.1016/j.ssc.2008.08.025

[20] Zhang, B.L., Wang, J. and Hou, Q. (2011) Molecular Dynamics Study of Helium Bubble Pressure in Titanium. Chinese Physics B, 20, Article ID: 036105.

https://doi.org/10.1088/1674-1056/20/3/036105 